\title{
A Qualitative Approach to Responsible Conduct of Research (RCR) Training Development: Identification of Metacognitive Strategies
}

\author{
Vykinta Kligyte $\cdot$ Richard T. Marcy • \\ Sydney T. Sevier · Elaine S. Godfrey • \\ Michael D. Mumford
}

Published online: 31 January 2008

(c) Springer Science+Business Media B.V. 2008

\section{Erratum to: Sci Eng Ethics \\ DOI 10.1007/s11948-007-9035-4}

Tables 1-3 in the article were not correctly published. Please find the corrected tables below.

The online version of the original article can be found under doi:10.1007/s11948-007-9035-4.

V. Kligyte $(\bowtie) \cdot$ R. T. Marcy · M. D. Mumford

Department of Psychology, University of Oklahoma, 455 W. Lindsey Street, Dale Hall Tower, Room 705, Norman, OK 73019-2007, USA

e-mail: vkligyte@psychology.ou.edu

R. T. Marcy

e-mail: rtmarcy@ou.edu

\section{D. Mumford}

e-mail: mmumford@ou.edu

\section{S. T. Sevier}

Department of Anthropology, University of Oklahoma, 455 W. Lindsey Street, Dale Hall Tower, Room 521, Norman, OK 73019-2007, USA

e-mail: ssevier@ou.edu

\section{E. S. Godfrey}

School of Meteorology, University of Oklahoma, 120 David L. Boren Blvd. Suite 5900, Norman, OK 73072-7307, USA

e-mail: esavageau@ou.edu 
Table 1 Summary of RCR training ${ }^{a}$

\begin{tabular}{|c|c|c|c|}
\hline Module & Title & Objectives & Content \\
\hline 1 & $\begin{array}{l}\text { Application of Ethical } \\
\text { Research Guidelines }\end{array}$ & $\begin{array}{l}\text { - Apply fundamental research } \\
\text { guidelines in ethical } \\
\text { decision-making }\end{array}$ & $\begin{array}{l}\text { - Guidelines Packet } \\
\text { - } 4 \text { Case Studies with } \\
\text { Questions }\end{array}$ \\
\hline 2 & $\begin{array}{l}\text { Complexity in Ethical } \\
\text { Decision-Making }\end{array}$ & $\begin{array}{l}\text { Discuss differences } \\
\text { between an ordinary } \\
\text { decision-making and } \\
\text { ethics-related decision- } \\
\text { making } \\
\text { - Discuss limitations of rule- } \\
\text { based approach }\end{array}$ & $\begin{array}{l}\text { - Self-Reflection Activity } \\
\text { - Pre-training Measures } \\
\text { - Module } 1 \text { Case Discussion }\end{array}$ \\
\hline 3 & $\begin{array}{l}\text { Personal Biases } \\
\text { Influencing Ethical } \\
\text { Decision-Making }\end{array}$ & $\begin{array}{l}\text { - Understand the existence of } \\
\text { cognitive biases } \\
\text { - Identify personal biases in } \\
\text { one's decision-making }\end{array}$ & $\begin{array}{l}\text { - Self-Enhancement } \\
\text { Demonstration } \\
\text { - Milgram's Study Video } \\
\text { - Behavior Predictions Activity }\end{array}$ \\
\hline 4 & $\begin{array}{l}\text { Common Reasoning } \\
\text { Errors Encountered in } \\
\text { Ethical Decision- } \\
\text { Making }\end{array}$ & $\begin{array}{l}\text { - Identify and generate } \\
\text { typical reasoning errors } \\
\text { that inhibit decision- } \\
\text { making }\end{array}$ & $\begin{array}{l}\text { - Identification of Common } \\
\text { Myths in Research Ethics } \\
\text { - Reasoning Error } \\
\text { Identification } \\
\text { - Reasoning Error Generation }\end{array}$ \\
\hline 5 & $\begin{array}{l}\text { Decision-Making } \\
\text { Strategies }\end{array}$ & $\begin{array}{l}\text { - Understand and apply } \\
\text { decision-making strategies } \\
\text { to address typical } \\
\text { reasoning errors }\end{array}$ & $\begin{array}{l}\text { - Case Analysis } \\
\text { - Strategy Generation } \\
\text { - Training Evaluation: Day } 1\end{array}$ \\
\hline 6 & $\begin{array}{l}\text { Field Specific } \\
\text { Differences }\end{array}$ & $\begin{array}{l}\text { - Locate one's field specific } \\
\text { guidelines } \\
\text { - Apply ethical decision- } \\
\text { making model utilizing } \\
\text { strategies }\end{array}$ & $\begin{array}{l}\text { - Locate Field Specific } \\
\text { Guidelines on the Web } \\
\text { - Two Case Analyses with } \\
\text { Questions }\end{array}$ \\
\hline 7 & $\begin{array}{l}\text { Sensemaking in } \\
\text { Ethical Decision- } \\
\text { Making }\end{array}$ & $\begin{array}{l}\text { - Interpret sensemaking } \\
\text { model and its importance } \\
\text { to ethical decision-making }\end{array}$ & $\begin{array}{l}\text { - Module } 6 \text { Homework Review } \\
\text { - Role Play Activity }\end{array}$ \\
\hline 8 & $\begin{array}{l}\text { Complex Field } \\
\text { Differences }\end{array}$ & $\begin{array}{l}\text { - Understand disparities in } \\
\text { ethical decision-making } \\
\text { across disciplines }\end{array}$ & $\begin{array}{l}\text { - Field Specific Guidelines } \\
\text { Review } \\
\text { - Group Case Analysis }\end{array}$ \\
\hline 9 & $\begin{array}{l}\text { Outcomes of } \\
\text { Different Perspectives }\end{array}$ & $\begin{array}{l}\text { - Make decisions while } \\
\text { considering multiple } \\
\text { perspectives (other } \\
\text { individuals, institutions, } \\
\text { etc.) }\end{array}$ & $\begin{array}{l}\text { - Case Analysis } \\
\text { - Role Play Activity }\end{array}$ \\
\hline 10 & $\begin{array}{l}\text { Key Components of } \\
\text { Ethical Decision- } \\
\text { Making }\end{array}$ & $\begin{array}{l}\text { - Rehearse key components } \\
\text { of ethical decision-making } \\
\text { - Apply acquired knowledge } \\
\text { in testing situations }\end{array}$ & $\begin{array}{l}\text { - Training Summary and Class } \\
\text { Discussion } \\
\text { - Training Evaluation: Day } 2 \\
\text { - Post-training and Personality } \\
\text { Measures }\end{array}$ \\
\hline
\end{tabular}

$\bar{a}$ Module 1 is self-directed pre-training; Modules 2 through 5 are presented in Day 1 classroom training. Module 6 is self-directed between training days; Modules 7 through 10 are presented in Day 2 classroom training. 


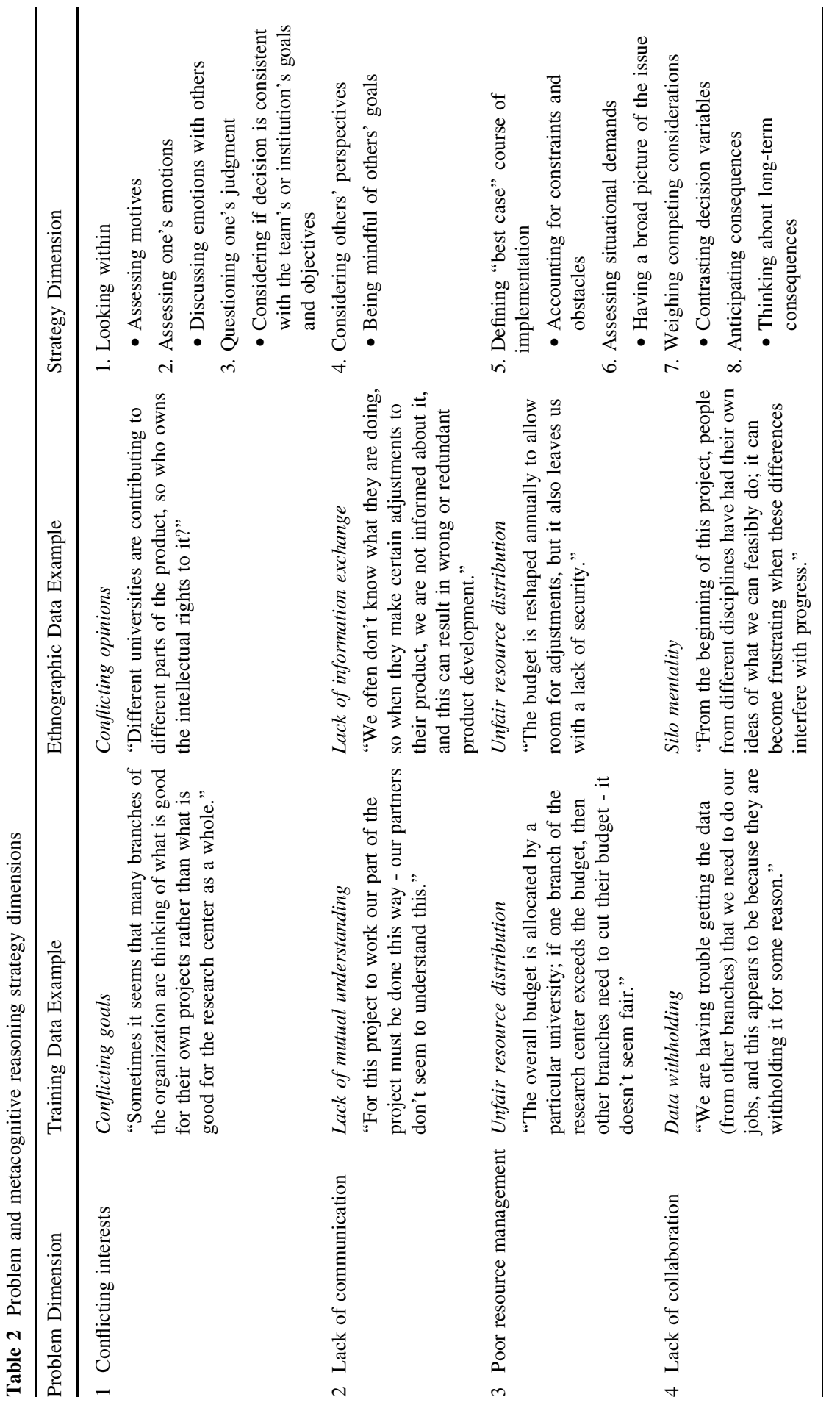



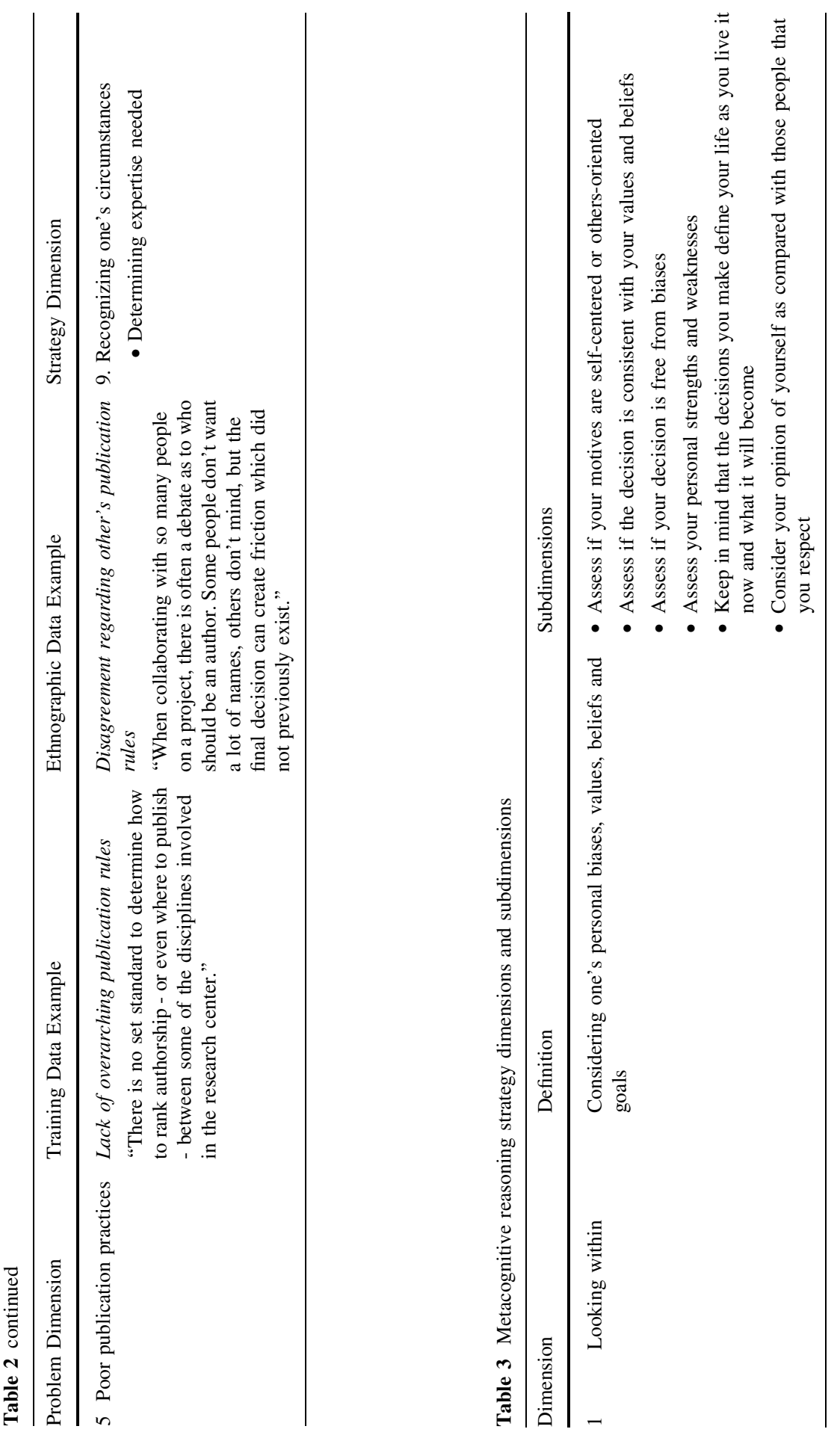


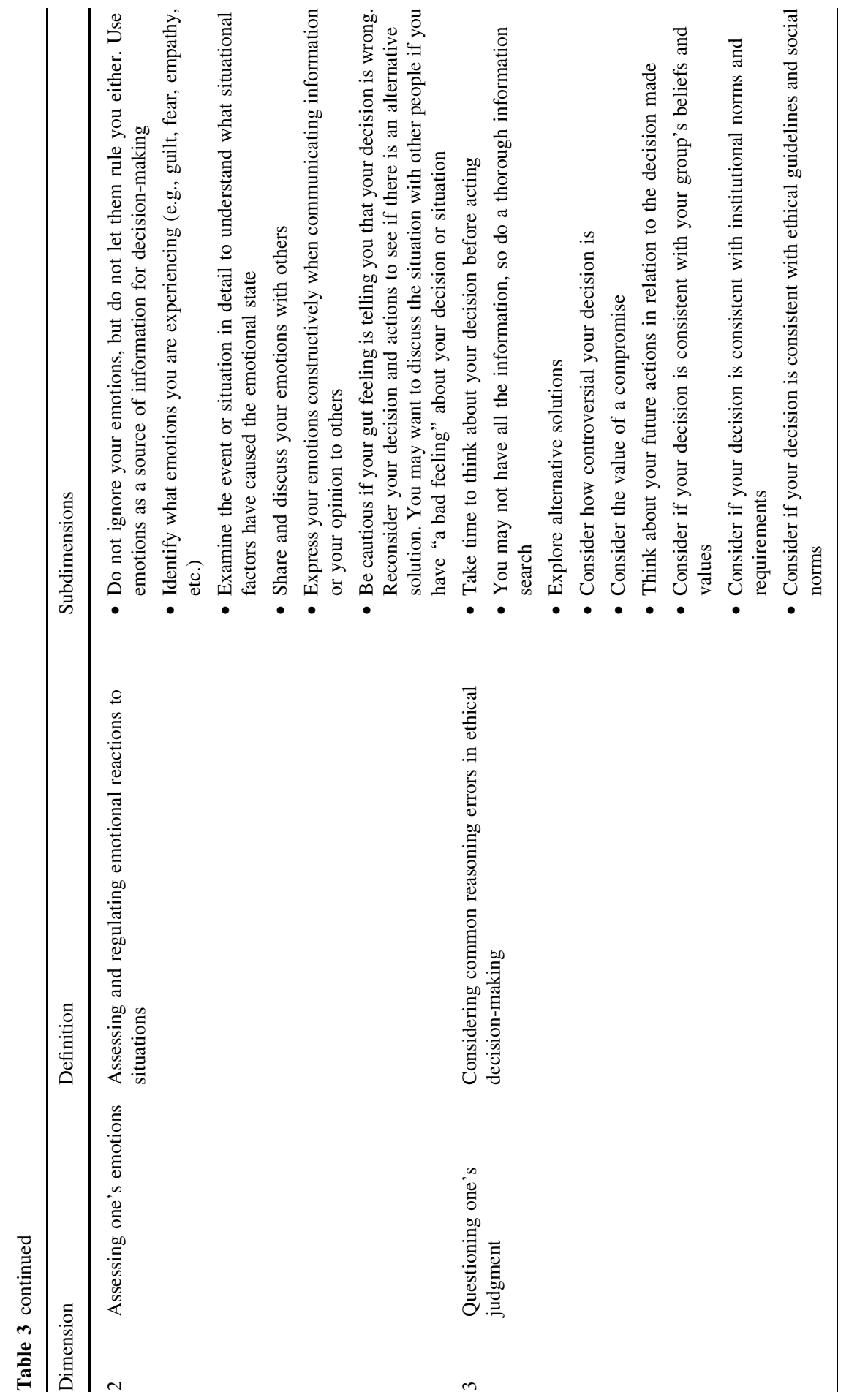




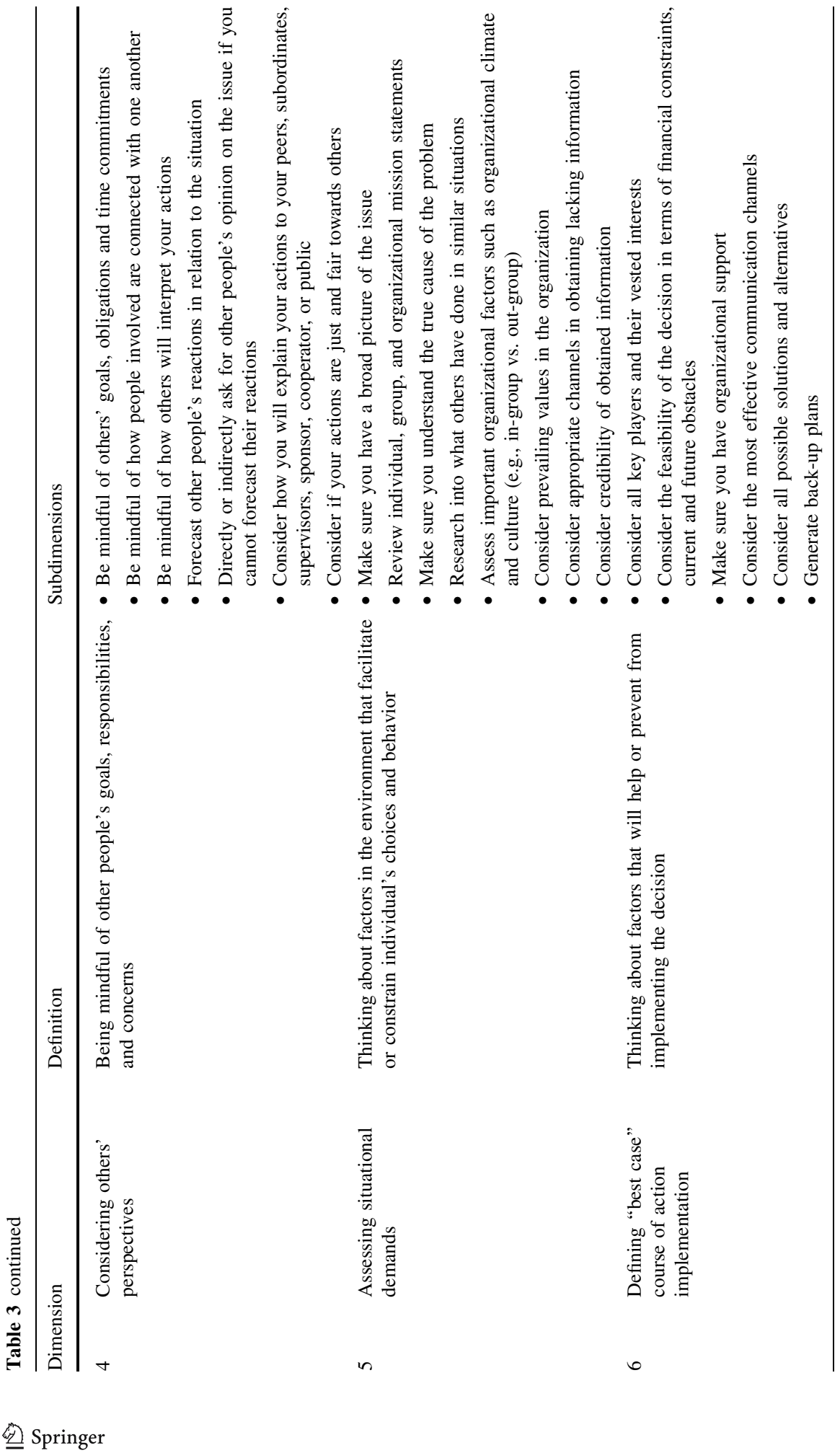




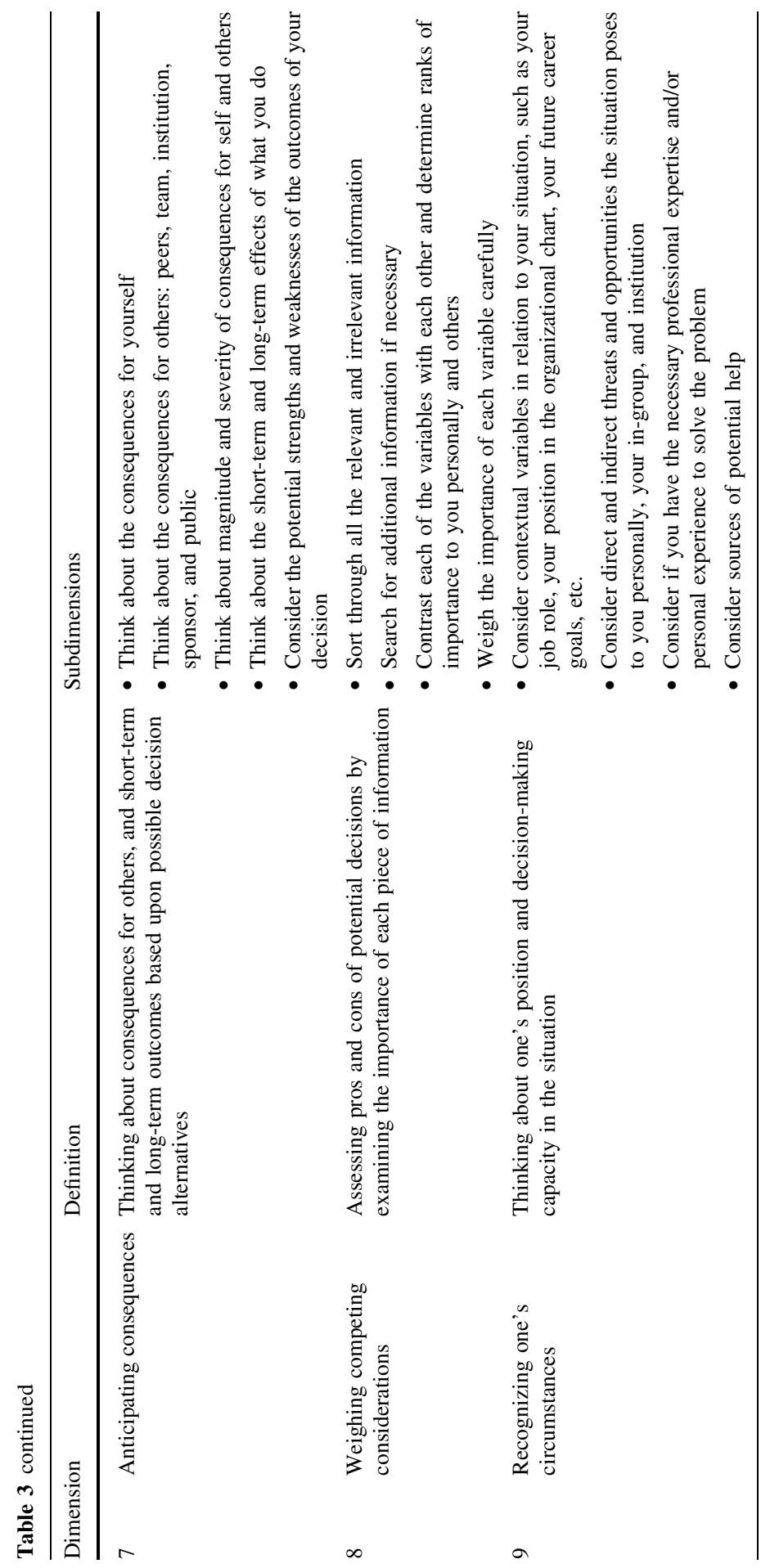

